\title{
Distributed Integrated Sliding Mode-Based Nonlinear Vehicle Platoon Control with Quadratic Spacing Policy
}

\author{
Lei Zuo (D), Ye Zhang, Maode Yan, and Wenrui Ma \\ Department of Electrical and Control Engineering, Chang'an University, Xi'an 710064, China \\ Correspondence should be addressed to Lei Zuo; 1_zuo@chd.edu.cn
}

Received 22 July 2020; Revised 11 November 2020; Accepted 9 December 2020; Published 28 December 2020

Academic Editor: Ning Wang

Copyright (c) 2020 Lei Zuo et al. This is an open access article distributed under the Creative Commons Attribution License, which permits unrestricted use, distribution, and reproduction in any medium, provided the original work is properly cited.

\begin{abstract}
This paper investigates the nonlinear vehicle platoon control problems with external disturbances. The quadratic spacing policy (QSP) is applied into the platoon control, in which the desired intervehicle distance is a quadratic function in terms of the vehicle's velocities. Comparing with the general constant time headway policy (CTHP), the QSP is more suitable to the human driving behaviors (HDB) and can improve the traffic capacity. Then, a novel platoon control scheme is proposed based on the distributed integrated sliding mode (DISM). Since the external disturbances are taken into consideration, the sliding mode method is employed to handle the disturbances. Moreover, the stability and string stability of the proposed platoon control system are strictly analyzed. In final, numerical simulations are provided to verify the proposed approaches.
\end{abstract}

\section{Introduction}

In recent years, the vehicle platoon control has received substantially increasing interests in the intelligent transportation system [1-3] due to its significant advantages in reducing traffic jams, improving the traffic safety, and increasing traffic capacity [4-6]. Generally, the objective of the vehicle platoon control is to drive a group of vehicles into a desired platoon from arbitrary initial positions. From this definition, we can find that the dynamics of vehicle, the communication topology, and the spacing policy play important roles in the vehicle platoon control.

To reach the desired platoon, many control theories have been applied to vehicle driving, including the consensus control [7], adaptive control [8-10], model predictive control [11], and the sliding mode control [12-17]. For example, a distributed consensus strategy with second-order dynamics is proposed to achieve the platooning of vehicles in [7], where the actuator saturation and absent velocity measurement are considered. In [8], the vehicle platoon control with velocity constraints, input saturations, and unknown driving resistances are handled by the adaptive neural sliding mode control techniques. By using the model predictive control method, a novel vehicle platoon control scheme with multiple constraints and communication delays is proposed in [11]. While considering the state of the leader and the braking force, a distributed integrated sliding mode-based platoon control algorithm is given in [12]. In these existing results, the sliding mode control method has attracted increasing interests due to its significant advantages in dealing with the external disturbances. For instance, the neuroadaptive quantized PID sliding mode control method for heterogeneous vehicle platoon is presented with external disturbances and unknown actuator dead-zone in [13]. The Pontryagin's minimum principle (PMP) based setpoint optimization and sliding mode control law are proposed for vehicle platoon in [14]. In [15], a distributed adaptive integrated sliding mode control method is developed to show the stability and string stability of the proposed vehicle platoon control system. Although these existing sliding mode control methods have great advantages in vehicle platoon control, the above studies mostly use the linear dynamics, which greatly limits their applications. Hence, it is necessary to further investigate the sliding modebased platoon control with nonlinear vehicles.

In addition, another key point of vehicle platoon is to select a reasonable intervehicle spacing strategy. It can improve the traffic capacity and reduce the road pressure [18]. 
Generally, there are two strategies for the desired platoon: the constant-spacing policy (CSP) and the variable-spacing policy (VSP). The differences between these spacing policies are the evolution policies of the desired distance between any adjacent vehicles [19]. Comparing with the CSP, the desired distances in VSP are usually time-varying and related to the vehicle's velocities. In detail, the constant time headway policy (CTHP) and the quadratic spacing policy (QSP) are two classical spacing policies in VSP. Particularly, the desired distance in QSP is a quadratic function in terms of the vehicle's velocities. It can meet the requirement of human driving behaviors (HDB). For example, the vehicle platoon control with QSP is proposed in [20], where the traffic flow stability and energy consumption are considered. In [21], a novel platoon control scheme with QSP is presented for the heavy trucks in mixed traffic situations, in which the fuel economy and pollution are taken into consideration. While considering the vehicle characteristics and road conditions, a vehicle platoon control algorithm with novel QSP is proposed for the longitudinal spacing control in [22]. Although the QSP shows great advantages in vehicle platoon control, there are few results applying the QSP into the vehicle platoon control with nonlinear dynamics.

Motivated by this fact, a distributed integrated sliding mode (DISM) based vehicle platoon control strategy with both QSP and nonlinear dynamics is proposed. The finitetime stability and string stability of the proposed vehicle platoon control system are strictly analyzed. In detail, a thirdorder nonlinear dynamics with external disturbances is applied to describe the vehicles. Then, the vehicle platoon with QSP is presented to maintain the desired intervehicle spacing. Since the QSP is a quadratic function in terms of the vehicle's velocities, the characteristic of this policy is more practical than the existing results with CTHP and CSP [23]. Moreover, the finite-time stability for each vehicle is shown by using the Lyapunov stability theory, and the string stability of the proposed vehicle platoon control system is demonstrated based on the coupled sliding mode method. In addition, an approximation function is employed to overcome the chattering in the sliding mode control.

The remainder of this paper is presented as follows. In Section 2, the preliminaries and problem formulation are presented. Then, the DISM-based vehicle platoon control with nonlinear dynamics and QSP is proposed, and the stability of proposed platoon control system is analyzed in Section 3. To illustrate the proposed approaches, numerical simulations are presented in Section 4. In final, Section 5 draws the conclusion and describes the future work.

Notations: throughout this paper, (1) R and $\mathrm{R}^{+}$represent the set of all real numbers and the set of all nonnegative real numbers, respectively. (2) $\|\cdot\|$ stands for the Euclidean norm of a vector. (3) $|\cdot|$ denotes the absolute value of real numbers. (4) The sgn(.) denotes the signum function.

\section{Preliminaries and Problem Formulation}

2.1. Vehicle Dynamics. Consider a group of nonlinear vehicles, which includes one leader and $N$ followers. Index these vehicles as $0,1, \ldots, N$, where 0 denotes the leader, and $1, \ldots, N$ are the followers. The dynamic model of each vehicle is shown as

$$
\left\{\begin{array}{l}
\dot{r}_{i}(t)=v_{i}(t) \\
\dot{v}_{i}(t)=a_{i}(t) \\
\dot{a}_{i}(t)=f_{i}\left(v_{i}(t), a_{i}(t)\right)+g_{i}\left(v_{i}(t)\right) b_{i}(t)+\omega_{i}(t)
\end{array}\right.
$$

where $a_{i}(t), v_{i}(t)$, and $r_{i}(t)$ are the acceleration, velocity, and position of the $i^{\text {th }}$ vehicle $(i=0,1,2, \ldots, N)$, respectively; $\omega_{i}(t)$ is the external disturbance of the $i^{\text {th }}$ vehicle, and $\left|\omega_{i}(t)\right| \leq \Omega ; b_{i}(t)$ is the engine control input; $f_{i}\left(v_{i}(t), a_{i}(t)\right)$, and $g_{i}\left(v_{i}(t)\right)$ are the nonlinear terms and are given as

$$
\left\{\begin{array}{l}
f_{i}\left(v_{i}(t), a_{i}(t)\right)=-\frac{1}{\zeta_{i}}\left(a_{i}(t)+\frac{\rho C_{i} d_{c i} v_{i}^{2}(t)}{2 m_{i}}+\frac{d_{m i}}{m_{i}}\right)-\frac{\rho C_{i} d_{c i} v_{i}(t) a_{i}(t)}{m_{i}} \\
g_{i}\left(v_{i}(t)\right)=\frac{1}{m_{i} \zeta_{i}}
\end{array}\right.
$$

where $m_{i}$ is the mass of the $i^{\text {th }}$ vehicle; $\rho$ is the mass density of the air; $d_{c i}$ is the coefficient of drag; $d_{m i}$ is the mechanical resistance; $C_{i}$ is the cross-section area of the $i^{\text {th }}$ vehicle; and $\zeta_{i}$ is the engine time.

Then, in order to solve the nonlinear vehicle dynamics of (1), a feedback linearization controller is introduced as follows:

$$
b_{i}(t)=u_{i}(t) m_{i}+\frac{\rho C_{i} d_{c i} v_{i}^{2}(t)}{2}+d_{m i}+\zeta_{i} \rho C_{i} d_{c i} v_{i}(t) a_{i}(t)
$$

Substituting (2) and (3) into (1), the model of the $i^{\text {th }}$ vehicle is described as

$$
\left\{\begin{array}{l}
\dot{r}_{i}(t)=v_{i}(t), \\
\dot{v}_{i}(t)=a_{i}(t), \\
\dot{a}_{i}(t)=-\frac{a_{i}(t)}{\zeta_{i}}+\frac{u_{i}(t)}{\zeta_{i}}+\omega_{i}(t),
\end{array}\right.
$$


where $u_{i}(t)$ denotes the control input for the $i^{\text {th }}$ vehicle after linearization.

Remark 1 . We use the real nonlinear vehicle dynamic model in this paper, and a feedback linearization method is deployed to transform the vehicle dynamic model into a third-order linear system. Comparing with the existing results, it is more similar to the real dynamics of a vehicle and more practical significance in vehicle platoon control.

For the communication topology, we have the following assumption.

Assumption 1. Suppose the communication topology among the vehicles is bidirectional. That is, each vehicle can only communicate with its adjacent neighbors.

2.2. Intervehicle Spacing Policy. The intervehicle spacing policy plays an important role in vehicle platoon control. For the intervehicle spacing policy, we usually select a proper function to construct the desired distance among the vehicles. The QSP is a classical spacing policy in vehicle platoon control. It is a quadratic function in terms of the vehicle's velocities, which is greatly suitable to the human driving behavior (HDB, $d_{i, i-1}=A+T V+G V^{2}$ ). The detailed QSP is shown by

$$
d_{i, i-1}=p_{0} v_{i}^{2}(t)+p_{1} v_{i}(t)+x,
$$

where $d_{i, i-1}$ denotes the desired distance between two adjacent vehicles; $x$ is the standstill spacing; and $p_{0}$ is the designed positive parameter and is given as $p_{0}=\left(\eta /\left(2 a_{\max }\right)\right)$, with $\eta$ being the safety factor depending on the road or weather, and $a_{\max }$ denoting the absolute value of the maximum possible deceleration. $p_{1}$ is the time delay in platoon systems, which compensates for the delay in braking or acceleration (about $10 \mathrm{~ms}-80 \mathrm{~ms}$ [23]).

Remark 2. Generally, the intervehicle spacing policy is closely related to the parameters $p_{0}$ and $p_{1}$. When $p_{0}, p_{1}>0$, the intervehicle spacing policy is QSP. In contrast, when $p_{0}=0, p_{1}>0$ and $p_{0}=0, p_{1}=0$, the intervehicle spacing policy reduces to a CTHP and a CSP, respectively, which have been studied extensively in vehicle platoon. Hence, comparing to the CTHP and CSP, the QSP is more general and practical. For the CSP, supposed that the distance of the adjacent vehicles maintained the same constant spacing, which may increase the traffic jams, the CTHP is also required to maintain a constant time headway parameter. Comparing with the general CSP and CTHP, the nonlinear QSP can be regarded as the VTHP to adjust the time headway and increase traffic capacity, which can also guarantee string stability and traffic flow stability. Hence, the vehicle platoon control with QSP is more practical in applications.
2.3. Problem Formulation. Based on the QSP, the spacing error $e_{i}(t)$ can be written as

$$
e_{i}(t)=\delta_{i}(t)-x-p_{0} v_{i}^{2}(t)-p_{1} v_{i}(t),
$$

where $\delta_{i}(t)=r_{i-1}(t)-r_{i}(t)-l$ denotes the distance between any two adjacent vehicles; $l$ is the length of each vehicle.

On this basis, we intend to design a DISM-based vehicle platoon control scheme with nonlinear dynamics and QSP. This paper has to meet the following requirements:

(1) Stability: the finite-time stability of each vehicle is guaranteed

(2) String stability [24]: the transient errors are not enlarging with vehicle index due to any maneuver of the lead vehicle, if the error propagation transfer function $G_{i}(s)$ satisfies

$$
\left|G_{i}(s)\right|=\left|\frac{E_{i+1}(s)}{E_{i}(s)}\right| \leq 1, \quad \text { for } i=1, \ldots, N,
$$

where $E_{i}(s)$ denotes the Laplace transform of $e_{i}(t)$

(3) For each vehicle, it must satisfy that

$$
\begin{aligned}
& v_{i}(t) \longrightarrow v_{0}(t), \\
& \delta_{i}(t) \longrightarrow x+p_{0} v_{0}^{2}(t)+p_{1} v_{0}(t), \quad \text { for } i=1, \ldots, N .
\end{aligned}
$$

\section{The DISM-Based Platoon Control Scheme}

In this section, a novel DISM-based vehicle platoon control scheme is proposed for a group of nonlinear vehicles with QSP. The finite-time stability of each vehicle and string stability of the proposed platoon control system are strictly analyzed.

First, the integrated sliding mode surface for the $i^{\text {th }}$ vehicle is shown as

$$
s_{i}(t)=\dot{e}_{i}(t)+\alpha_{1} e_{i}(t)+\alpha_{2} \int_{0}^{t} e_{i}(\tau) \mathrm{d} \tau,
$$

where $\alpha_{1}$ and $\alpha_{2}$ are the positive constants.

According to Hurwitz stability theory, $\alpha_{1}$ and $\alpha_{2}$ are required to make all the eigenvalues of $p^{2}+\alpha_{1} p+\alpha_{2}=0$ contain a real-negative part. In this case, let $\alpha_{1}=2 \epsilon$ and $\alpha_{2}=\epsilon^{2}$, where $\epsilon$ is a positive constant [25].

Since the sliding mode surface in (9) cannot guarantee the string stability of the vehicle platoon, an improved coupled sliding mode surface is provided as follows:

$$
S_{i}(t)= \begin{cases}s_{i+1}(t)-\beta s_{i}(t), & i=1, \ldots, N-1, \\ -\beta s_{i}(t), & i=N,\end{cases}
$$


where $\beta$ is the parameter of coupled intensity satisfying $0<\beta \leq 1$. The relationship between $S_{i}(t)$ and $s_{i}(t)$ is shown as

$$
S(t)=B s(t)
$$

where $s(t)=\left[\begin{array}{llll}s_{1}(t) & s_{2}(t) & \ldots & s_{N}(t)\end{array}\right]^{T}, S(t)=\left[\begin{array}{lll}S_{1}(t) & S_{2}(\end{array}\right.$ t). $\left.S_{N}(t)\right]^{T}$, and

$$
B=\left[\begin{array}{ccccc}
-\beta & 1 & \cdots & 0 & 0 \\
0 & -\beta & 1 & \cdots & 0 \\
\vdots & \vdots & \ddots & \vdots & \vdots \\
0 & 0 & \cdots & -\beta & 1 \\
0 & 0 & \cdots & 0 & -\beta
\end{array}\right] .
$$

To further show the relationship between $s_{i}$ and $S_{i}$, we have the following lemmas.

Lemma 1. Equivalence of the convergence of the CSS and each sliding surface toward zero [26]: $S_{i}$ becomes zero for all $i=1, \ldots, N$, if and only if $s_{i}$ becomes zero.

According to (6), (9), and (10), $\dot{S}_{i}$ is presented as

$$
\begin{aligned}
\dot{S}_{i}(t) & =\dot{s}_{i+1}-\beta \dot{s}_{i}, \quad i=1,2, \ldots, N-1, \\
& =\left[\ddot{e}_{i+1}(t)+\alpha_{1} \dot{e}_{i+1}+\alpha_{2} e_{i+1}(t)\right]-\beta\left[\ddot{e}_{i}(t)+\alpha_{1} \dot{e}_{i}(t)+\alpha_{2} e_{i}(t)\right] \\
& =-\beta\left(\left[a_{i-1}(t)-a_{i}(t)\right]-\phi_{i}(t)\left[-\frac{a_{i}(t)}{\zeta_{i}}+\frac{u_{i}(t)}{\zeta_{i}}+\omega_{i}(t)\right]-2 p_{0} a_{i}^{2}(t)\right)+D_{i}(t) \\
& =\beta \phi_{i}(t)\left[-\frac{a_{i}(t)}{\zeta_{i}}+\frac{u_{i}(t)}{\zeta_{i}}+\omega_{i}(t)\right]-\beta\left[a_{i-1}(t)-a_{i}(t)\right]+2 \beta p_{0} a_{i}^{2}(t)+D_{i}(t),
\end{aligned}
$$

where $\phi_{i}(t) \triangleq 2 p_{0} v_{i}(t)+p_{1}$, and $D_{i}(t)=\left[\ddot{e}_{i+1}(t)+\alpha_{1} \dot{e}_{i+1}(t)\right.$

For $i=N$, we obtain that $\left.+\alpha_{2} e_{i+1}(t)\right]-\beta\left[\alpha_{1} \dot{e}_{i}(t)+\alpha_{2} e_{i}(t)\right]$

$$
\begin{aligned}
\dot{S}_{N}(t) & =-\beta \dot{s}_{N} \\
& =-\beta\left[\ddot{e}_{N}(t)+\alpha_{1} \dot{e}_{N}(t)+\alpha_{2} e_{N}(t)\right] \\
& =-\beta\left(\left[a_{N-1}(t)-a_{N}(t)\right]-\phi_{N}(t)\left[-\frac{a_{N}(t)}{\zeta_{N}}+\frac{u_{N}(t)}{\zeta_{N}}+\omega_{N}(t)\right]-2 p_{0} a_{N}^{2}(t)\right)+D_{N}(t) \\
& =\beta \phi_{N}(t)\left[-\frac{a_{N}(t)}{\zeta_{N}}+\frac{u_{N}(t)}{\zeta_{N}}+\omega_{N}(t)\right]-\beta\left[a_{N-1}(t)-a_{N}(t)\right]+2 \beta p_{0} a_{N}^{2}(t)+D_{N}(t),
\end{aligned}
$$

where $\phi_{N}(t) \triangleq 2 p_{0} v_{N}(t)+p_{1}$, and $D_{N}(t)=-\beta\left[\alpha_{1} \dot{e}_{N}(t)+\right.$ $\left.\alpha_{2} e_{N}(t)\right]$.

Then, the following two lemmas are provided to show the finite-time stability and string stability.

Lemma 2. (Barbalat Lemma [27]). If $\phi(t): R \longrightarrow R^{+}$is a uniformly continuous function for $t \geq 0$ and the limit of the integral $\lim _{t \rightarrow \infty} \int_{0}^{t} \phi(\tau) d \tau$ exists and is finite, then

$$
\lim _{t \longrightarrow \infty} \phi(t)=0 \text {. }
$$

Lemma 3. (Finite-Time Stability [28]). Suppose there is a positive definite Lyapunov function $V(x, t)$ defined on $U \times R^{+}$, where $U$ is the neighborhood of the origin. There are positive real constants $c>0$ and $a \in(0,1)$, such that $\dot{V}(x, t)+$ $c V^{a}(x, t)$ is negative semidefinite on $U$. Then, $V(x, t)$ is locally finite-time convergent. In addition, the settling time $T$ satisfies $T \leq\left(V^{1-a}\left(x_{0}, t\right)\right) /(c(1-a))$ for any given initial condition $x\left(t_{0}\right)$ in the neighborhood of the origin in $U$.

Then, we have the following DISM-based vehicle platoon control schemes. 


$$
u_{i}(t)=-\frac{1}{\beta \phi_{i}(t)}\left\{-\beta \zeta_{i}\left[a_{i-1}(t)-a_{i}(t)\right]+\zeta_{i} D_{i}(t)+2 \beta \zeta_{i} p_{0} a_{i}^{2}(t)+\zeta_{i} \gamma \operatorname{sgn}\left(S_{i}(t)\right)\right\}+a_{i}(t), \quad i=1,2, \ldots, N,
$$

with

$$
D_{i}(t)= \begin{cases}{\left[\ddot{e}_{i+1}(t)+\alpha_{1} \dot{e}_{i+1}(t)+\alpha_{2} e_{i+1}(t)\right]-\beta\left[\alpha_{1} \dot{e}_{i}(t)+\alpha_{2} e_{i}(t)\right],} & i=1, \ldots, N-1, \\ -\beta\left[\alpha_{1} \dot{e}_{N}(t)+\alpha_{2} e_{N}(t)\right], & i=N,\end{cases}
$$

where $\phi_{i}(t) \triangleq 2 p_{0} v_{i}(t)+p_{1}$; according to the velocity of each vehicle bound, $\left|\phi_{i}(t)\right| \leq \Phi \cdot \gamma$ is the positive design parameter of the controller and satisfies that $\gamma>\beta \Omega \Phi$.

To formally show the finite-time stability of each vehicle and string stability of the whole vehicle platoon, the following theorems are proposed.

Theorem 1. Consider a vehicle platoon system described by (4). The communication topology among vehicles is bidirectional, and the QSP is shown in (5). Then, by using the proposed platoon control law in (16), the finite-time stability of each vehicle can be guaranteed.

Proof. Consider the following Lyapunov function candidate:

$$
V(t)=\sum_{i=1}^{n} V(t)_{i}=\sum_{i=1}^{n} \frac{1}{2} S_{i}^{2}(t)
$$

Then, taking the derivation of $V_{i}(t)$, we obtain that

$$
\dot{V}(t)=\sum_{i=1}^{n} S_{i}(t) \dot{S}_{i}(t)
$$

Substituting (13)-(16) into (19), it follows

$$
\begin{aligned}
\dot{V}(t)= & \sum_{i=1}^{n}\left\{S _ { i } ( t ) \left[\beta \phi_{i}(t)\left(-\frac{a_{i}(t)}{\zeta_{i}}+\frac{u_{i}(t)}{\zeta_{i}}+\omega_{i}(t)\right)\right.\right. \\
& \left.\left.-\beta\left(a_{i-1}(t)-a_{i}(t)\right)+2 \beta p_{0} a_{i}^{2}(t)+D_{i}(t)\right]\right\} \\
= & \sum_{i=1}^{n}\left\{S_{i}(t)\left[-\gamma \operatorname{sgn}\left(S_{i}(t)\right)+\beta \phi_{i}(t) \omega_{i}(t)\right\}\right. \\
= & \sum_{i=1}^{n}\left\{-\gamma \operatorname{sgn}\left(S_{i}(t)\right) S_{i}(t)+\beta \phi_{i}(t) \omega_{i}(t) S_{i}(t)\right\} \\
\leq & \sum_{i=1}^{n}\left\{-\gamma\left|S_{i}(t)\right|+\beta \Phi \Omega\left|S_{i}(t)\right|\right\} \\
= & \sum_{i=1}^{n}\left\{-[\gamma-\beta \Phi \Omega]\left|S_{i}(t)\right|\right\} \leq 0 .
\end{aligned}
$$

Denoting $\varphi(t) \triangleq[\gamma-\beta \Phi \Omega] \sum_{i=1}^{n}\left|S_{i}(t)\right|$ and integrating it from 0 to $t$, we have that

$$
V(0)-V(t) \geq \int_{0}^{t} \varphi(\tau) \mathrm{d} \tau
$$

Since $\dot{V}(t) \leq 0$, we have that $V(0)-V(t) \geq 0$ is positive and bounded if $V(0)$ is bounded. Then, according to Lemma 2, we obtain that

$$
\lim _{t \longrightarrow \infty} \varphi(t)=\lim _{t \longrightarrow \infty}[\gamma-\Phi \beta \Omega] \sum_{i=1}^{n}\left|S_{i}(t)\right|=0
$$

As $\gamma-\Phi \beta \Omega>0$, it follows that $\lim _{t \rightarrow \infty} S_{i}(t)=0$. Then, according to (9), (11), and Lemma $1, s_{i}(t)$ and $e_{i}(t)$ would converge to zeros. Furthermore, as $\gamma-\Phi \beta \Omega \geq \bar{\gamma}>0$, (20) can also be written as

$$
\dot{V}(t) \leq-[\gamma-\beta \Phi \Omega] \sum_{i=1}^{n}\left|S_{i}(t)\right| \leq-\bar{\gamma} \sqrt{2 V(t)},
$$

and equivalently $\dot{V}(t)+\bar{\gamma} \sqrt{2 V(t)} \leq 0$, where $\sqrt{2 V(t)}$ satisfying $\sqrt{2 V(t)} \leq \sqrt{2 V(0)}-\bar{\gamma} t$. Then, we can get that $V(t)=0$ and equivalently $S_{i}(t)=0$ when $T \geq(\sqrt{2 V(0)} / \bar{\gamma})$ by Lemma 3 . It indicates that $s_{i}(t)$ and spacing error $e_{i}(t)$ converge to zero in a finite time. On this basis, the finite-time stability of each vehicle is guaranteed.

Theorem 2. Since $0<\beta \leq 1$ and $S_{i}(t) \longrightarrow 0$ in finite time, then the string stability of whole vehicle platoon system is guaranteed.

Proof. Since $S_{i}(t) \longrightarrow 0$ in a finite time, $s_{i+1}(t)-\beta s_{i}(t)=0$. Then, we can obtain that

$$
\beta\left(\dot{e}_{i}(t)+\alpha_{1} e_{i}(t)+\alpha_{2} \int_{0}^{t} e_{i}(\tau) \mathrm{d} \tau\right)=\dot{e}_{i+1}(t)+\alpha_{1} e_{i+1}(t)+\alpha_{2} \int_{0}^{t} e_{i+1}(\tau) \mathrm{d} \tau
$$


Since $\dot{e}_{i}(0)=0, \quad e_{i}(0)=0$, and $e_{i}^{-1} \triangleq \int_{-\infty}^{0} e_{i}(t) \mathrm{d} t=0$, take the Laplace transform of (24) and obtain that

$$
\beta\left(s+\alpha_{1}+\frac{\alpha_{2}}{s}\right) E_{i}(s)=\left(s+\alpha_{1}+\frac{\alpha_{2}}{s}\right) E_{i+1}(s) .
$$

According to the condition $0<\beta \leq 1$, we obtain that

$$
\left|G_{i}(s)\right|=\left|\frac{E_{i+1}(s)}{E_{i}(s)}\right|=|\beta| \leq 1 \text {. }
$$

From (26), the string stability of the vehicle platoon can be achieved.
Remark 3. Since we have employed the signum function $\operatorname{sgn}\left(S_{i}(t)\right)$ in (16), it may cause chattering in practical applications. In this case, a sigmoid-like function $\left(S_{i}(t) /\left(\left|S_{i}(t)\right|+\sigma\right)\right)$ is employed to eliminate this chattering. In addition, the $\sigma$ in this sigmoid-like function should be small enough. It is because when a large $\sigma$ is chosen, it would lead to a less accurate control result. Thus, we should trade-off the relationship between the value of $\sigma$ and the control accuracy in practical applications.

Invoking the sigmoid-like function $\left(S_{i}(t) /\left(\left|S_{i}(t)\right|+\sigma\right)\right)$, $u_{i}(t)$ can be rewritten as

$$
u_{i}(t)=-\frac{1}{\beta \phi_{i}(t)}\left\{-\beta \zeta_{i}\left[a_{i-1}(t)-a_{i}(t)\right]+\zeta_{i} D_{i}(t)+2 \beta p_{0} a_{i}^{2}(t) \zeta_{i}\right\}+\frac{\zeta_{i} \gamma}{\beta \phi_{i}(t)} \frac{S_{i}(t)}{\left|S_{i}(t)\right|+\sigma}+a_{i}(t), \quad i=1,2, \ldots, N
$$

Based on the above theorems and analyses, the following algorithm is provided to illustrate the proposed vehicle platoon scheme.

In Algorithm 1, it is worth noting that the initial spacing errors and the initial velocity errors of all vehicles are required to be zero, simultaneously. Furthermore, to facilitate the adjustment of parameters $p_{0}$ and $p_{1}$, it is better to choose some small initial velocities for all the vehicles. Since the variable range, the time headway is limited [20], the parameters $p_{0}$ and $p_{1}$ are also limited by $0<p_{0}, p_{1} \leq 1$. Then, in order to guarantee the string stability, the $\beta$ must satisfy that $0<\beta \leq 1$.

\section{Numerical Simulation}

In this section, the numerical simulations are provided for a platoon with 4 followers and one leader to verify the proposed approaches.

The initial states of the leader are set as $r_{0}=0 \mathrm{~m}$ and $v_{0}=2 \mathrm{~m} / \mathrm{s}$, and the evolution of leader's velocity is shown as

$$
v_{0}(t)= \begin{cases}2 \mathrm{~m} / \mathrm{s}, & 0 \mathrm{~s}<t \leq 3 \mathrm{~s}, \\ 2+2(t-3) \mathrm{m} / \mathrm{s}, & 3 \mathrm{~s}<t \leq 5 \mathrm{~s} \\ 6 \mathrm{~m} / \mathrm{s}, & 5 \mathrm{~s}<t \leq 10 \mathrm{~s} \\ 6-2(t-10) \mathrm{m} / \mathrm{s}, & 10 \mathrm{~s}<t \leq 12 \mathrm{~s} \\ 2 \mathrm{~m} / \mathrm{s}, & 12 \mathrm{~s}<t \leq 18 \mathrm{~s} \\ 2+(t-18) \mathrm{m} / \mathrm{s}, & 18 \mathrm{~s}<t \leq 20 \mathrm{~s}, \\ 4 \mathrm{~m} / \mathrm{s}, & 20 \mathrm{~s}<t \leq 25 \mathrm{~s} \\ 4-(t-25) \mathrm{m} / \mathrm{s}, & 25 \mathrm{~s}<t \leq 27 \mathrm{~s} \\ 2 \mathrm{~m} / \mathrm{s}, & 27 \mathrm{~s}<t \leq 60 \mathrm{~s} .\end{cases}
$$

The initial positions and initial velocities of the followers are set as $x(0)=[-24.76,-49.52,-74.28,-99.04]$ and $v(0)=[2,2,2,2]$, respectively. All controller's parameters are set as $\gamma=1.5, \beta=0.6, \alpha_{1}=2, \alpha_{2}=1, p_{0}=0.155$, $p_{1}=0.07, \sigma=0.02$, and $\zeta=0.3$. Then, the standstill distance is given as $x=18 \mathrm{~m}$, and the length of each vehicle is $l=6 \mathrm{~m}$.
The external disturbance of the $i^{\text {th }}$ vehicle is given by $w_{i}(t)=0.003 \sin (2 \pi t)$.

On this basis, the simulation results of proposed vehicle platoon control scheme are shown as follows.

Figure 1 illustrates the positions and velocities of all vehicles. As shown in Figure 1, there is no collision between any two adjacent vehicles during the steady-state condition and the initial transient. Then, followers track the velocity of lead vehicle, in which the velocities of followers converge to the velocity of leader $(2 \mathrm{~m} / \mathrm{s})$. The convergence time of the velocities is about $35 \mathrm{~s}$.

The distances and spacing errors of the platoon with QSP are illustrated in Figure 2. As shown in this figure, the distances of all adjacent vehicles converge a constant value $(18.5 \mathrm{~m})$. Meanwhile, the spacing errors converge to zero in a finite time, and the string stability of the vehicle platoon is guaranteed. From Figures 1 and 2, it can be obtained that all vehicles satisfy the control objective in (8). The proposed vehicle platoon system is stable and reaches the desired formation under the DISM control scheme with QSP.

The sliding mode surface is shown in Figure 3. From Figure 3, it can be observed that the $S_{i}(t)$ reaches the sliding mode surface $S_{i}(t)=0$ in a finite time and there is no chattering.

The distances and spacing errors of the platoon with CTHP are presented in Figure 4. From Figure 4, it can be seen that the distance between any two adjacent vehicles converges to a constant value $(20 \mathrm{~m})$ and the spacing errors converge to zero in a finite time. Then, in order to show the advantage of the vehicle platoon control with QSP, we compared our proposed approaches in Figure 3 with the vehicle platoon with CTHP in Figure 4; the platoon with QSP has smaller intervehicle distance than the platoon with CTHP. Apparently, the platoon with QSP can provide greater traffic capacity. Hence, the platoon with QSP is superior to the platoon with CTHP. The simulation results validate the proposed approaches.

According to the vehicle platoon performance index in [29], Table 1 shows the simulation results of different spacing policies in tracking performance, fuel economy, and ride 
Input: the initial position, velocity, acceleration of each vehicle;

The controller parameters;

Output: the spacing error of the any two adjacent vehicles converges to zero;

(1) Initialization: the initial positions, velocities, accelerations of one lead vehicle and 4 followers: $r_{i}(0), v_{i}(0), a_{i}(0)$; Controller parameters: $p_{0}, p_{1}, x, l, \beta, \alpha_{1}, \alpha_{2}, \zeta_{i}, \gamma$

(2) for $t=t_{0}: \Delta t: t_{f}$ do

(3) calculate $e_{i}(t), \dot{e}_{i}(t), \ddot{e}_{i}(t)$ by using (6);

(4) construct integrated sliding surface $s_{i}(t)$ by employing (9);

(5) calculate $S_{i}(t)$ and $D_{i}$ based on (10), (13), (14);

(6) according to (16), calculate $u_{i}(t)$;

(7) update the acceleration, velocity, and position information of the $i^{\text {th }}$ vehicle respectively by using (4);

(8) end for

Algorithm 1: The DISM-based platoon control algorithm with QSP.

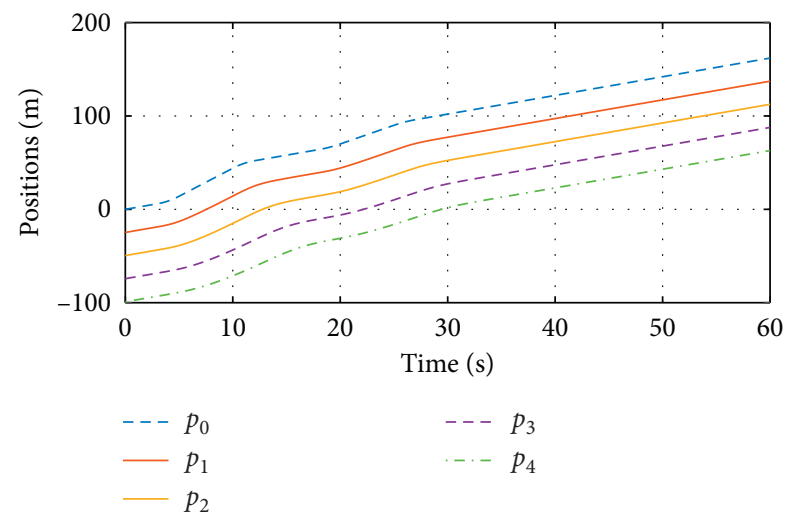

(a)

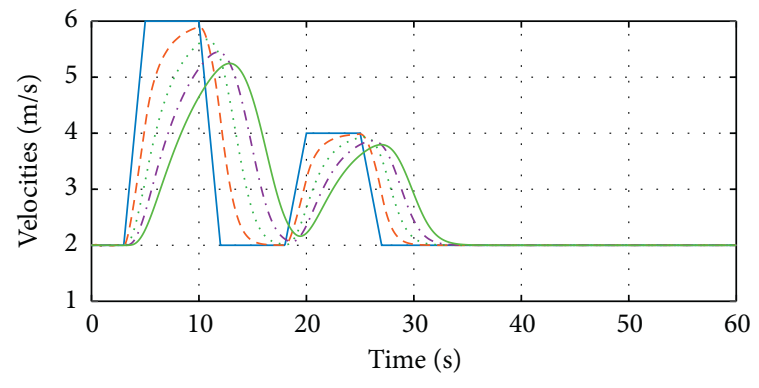

$\begin{array}{cc}- & u_{0} \\ --- & u_{1} \\ \ldots \ldots & u_{2}\end{array}$

$-u_{3}$

(b)

FIgURE 1: Positions and velocities of vehicles.

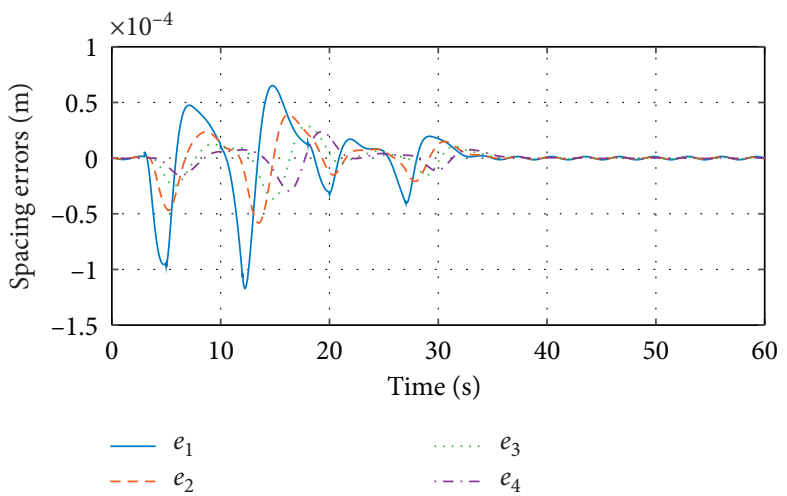

(a)

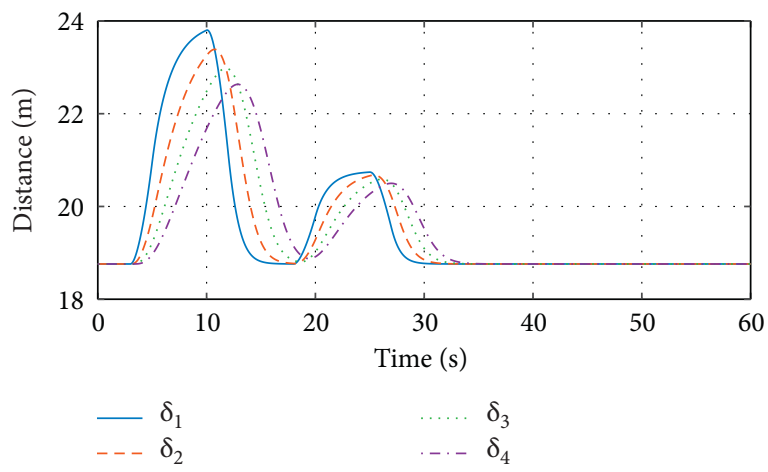

(b)

FIGURE 2: Spacing errors and distances of the platoon.

comfort. As shown in Table 1, the fuel economy and ride comfort of the platoon with the QSP are better, and the platoon with the CTHP has better tracking performance. In detail, the energy consumption and comfort ride of the vehicle platoon is mainly caused by unnecessary braking or acceleration. Comparing with the CTHP, the QSP can be regarded as the variable time headway spacing policy; thus, the energy consumption value of the vehicle platoon with QSP is smaller. Therefore, the QSP can reduce the fuel consumption and enhance ride comfort. 


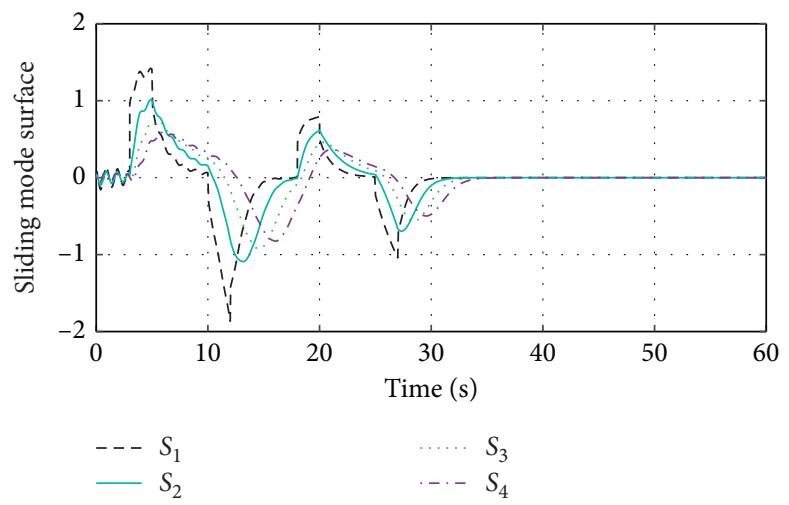

FIgURE 3: Sliding mode surface.

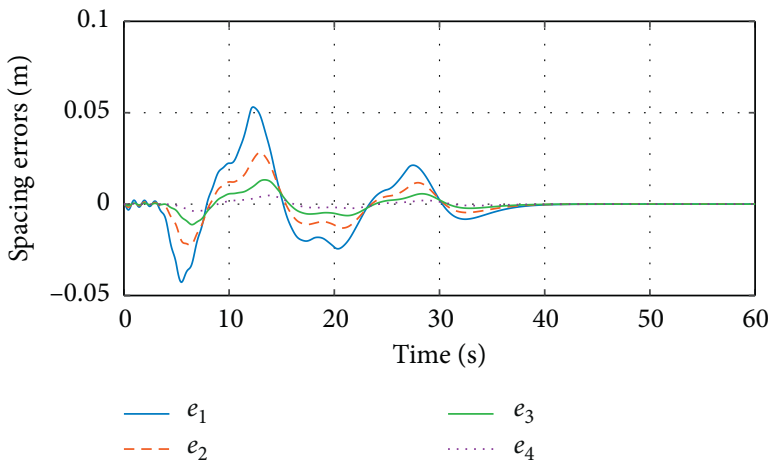

(a)

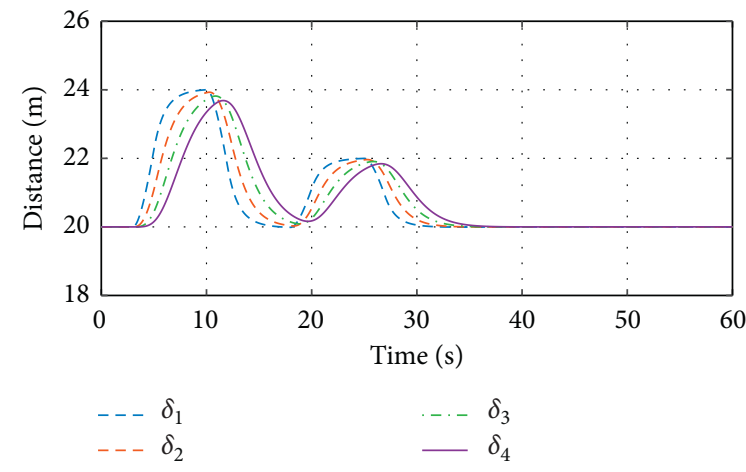

(b)

FIGURE 4: Distances and spacing errors of the platoon with CTHP $\left(h_{i}=1\right)$ [25].

TABLE 1: The performance comparison of different spacing policies.

\begin{tabular}{lcccccc}
\hline \multirow{2}{*}{$\begin{array}{l}\text { Vehicle } \\
\text { index }\end{array}$} & \multicolumn{2}{c}{$\begin{array}{c}\text { Tracking } \\
\text { performance }\end{array}$} & \multicolumn{2}{c}{ Fuel economy } & \multicolumn{2}{c}{ Ride comfort } \\
& CTH & QSP & CTH & QSP & CTH & QSP \\
\hline 1 & 11.1820 & 15.3432 & 12.2174 & 10.6983 & 0.8967 & 0.7205 \\
2 & 8.1891 & 10.1266 & 8.5112 & 7.1400 & 0.3128 & 0.2319 \\
3 & 6.5083 & 7.4740 & 6.6575 & 5.4629 & 0.1689 & 0.1295 \\
4 & 5.3897 & 5.8381 & 5.4872 & 4.4124 & 0.1102 & 0.0923 \\
\hline
\end{tabular}

\section{Conclusion}

In this paper, we study the nonlinear vehicle platoon problems with external disturbances, in which the QSP is applied into the platoon control. The desired intervehicle distance in the QSP is a quadratic function in terms of the vehicle's velocities. Comparing with CTHP, the QSP can be regarded as the VTHP to improve traffic capacity and reduce energy consumption. Then, a novel platoon control scheme based on the DISM with QSP and nonlinear dynamics is proposed. Moreover, the finite-time stability of each vehicle is shown by using the Lyapunov theory, and a coupled sliding mode surface is adopted to guarantee the string stability of the vehicle platoon system. In final, the numerical simulations are provided to verify the proposed approaches. In the future, we will continue to investigate the vehicle platoon with VSP where the unknown external disturbances and mixed traffic situations will be considered.

\section{Data Availability}

The data used to support the findings of this study are included within the article and are available from the corresponding author upon request.

\section{Conflicts of Interest}

The authors declare that there are no conflicts of interest.

\section{Acknowledgments}

This work was supported by the National Natural Science Foundation of China (Nos.51909008, 61803040, and 62003054) and the Natural Science Basic Research Plan in Shannxi Province of China (Nos.2019GY-218 and 2020GY113).

\section{References}

[1] X. Hu, C. Zou, X. Tang, T. Liu, and L. Hu, "Cost-optimal energy management of hybrid electric vehicles using fuel cell/ battery health-aware predictive control," IEEE Transactions on Power Electronics, vol. 35, no. 1, pp. 382-392, 2020. 
[2] G. Guo and D. Li, "Adaptive sliding mode control of vehicular platoons with prescribed tracking performance," IEEE Transactions on Vehicular Technology, vol. 68, no. 8, pp. 7511-7520, 2019.

[3] H. Guo, Z. Yin, D. Cao, H. Chen, and C. Lv, "A review of estimation for vehicle tire-road interactions toward automated driving," IEEE Transactions on Systems, Man, and Cybernetics: Systems, vol. 49, no. 1, pp. 14-30, 2019.

[4] H. Guo, F. Liu, F. Xu, H. Chen, D. Cao, and Y. Ji, "Nonlinear model predictive lateral stability control of active chassis for intelligent vehicles and its fpga implementation," IEEE Transactions on Systems, Man, and Cybernetics: Systems, vol. 49, no. 1, pp. 2-13, 2019.

[5] T. Liu, X. Tang, H. Wang, H. Yu, and X. Hu, "Adaptive hierarchical energy management design for a plug-in hybrid electric vehicle," IEEE Transactions on Vehicular Technology, vol. 68, no. 12, pp. 11513-11 522, 2019.

[6] X. Hu, T. Liu, X. Qi, and M. Barth, "Reinforcement learning for hybrid and plug-in hybrid electric vehicle energy management: recent advances and prospects," IEEE Industrial Electronics Magazine, vol. 13, no. 3, pp. 16-25, 2019.

[7] P. Yang, Y. Tang, M. Yan, and X. Zhu, "Consensus based control algorithm for nonlinear vehicle platoons in the presence of time delay," International Journal of Control, Automation and Systems, vol. 17, no. 3, pp. 752-764, 2019.

[8] M. Yan, J. Song, P. Yang, and L. Zuo, "Neural adaptive slidingmode control of a bidirectional vehicle platoon with velocity constraints and input saturation," Complexity, vol. 2018, Article ID 1696851, 16 pages, 2018.

[9] J. Song, M. Yan, Y. Ju, and P. Yang, "Nonlinear gain feedback adaptive dsc for a class of uncertain nonlinear systems with asymptotic output tracking," Nonlinear Dynamics, vol. 98, no. 3, pp. 2195-2210, 2019.

[10] J. Song, M. Yan, and P. Yang, "Neural adaptive dynamic surface asymptotic tracking control for a class of uncertain nonlinear system," Circuits, Systems, and Signal Processing, vol. 12, pp. 1-26, 2020.

[11] M. Yan, W. Ma, L. Zuo, and P. Yang, "Distributed model predictive control for platooning of heterogeneous vehicles with multiple constraints and communication delays," Journal of Advanced Transportation, vol. 2020, pp. 1-16, Article ID 4657584, 2020.

[12] Y. Li, C. Tang, S. Peeta, and Y. Wang, "Integral-sliding-mode braking control for a connected vehicle platoon: theory and application," IEEE Transactions on Industrial Electronics, vol. 66, no. 6, pp. 4618-4628, 2019.

[13] X. Guo, J. Wang, F. Liao, and R. S. H. Teo, "Neuroadaptive quantized pid sliding-mode control for heterogeneous vehicular platoon with unknown actuator deadzone," International Journal of Robust and Nonlinear Control, vol. 29, no. 1, pp. 188-208, 2019.

[14] G. Guo and D. Li, "Pmp-based set-point optimization and sliding-mode control of vehicular platoons," IEEE Transactions on Computational Social Systems, vol. 5, no. 2, pp. 553-562, 2018.

[15] X. Guo, J. Wang, F. Liao, and R. S. H. Teo, "Distributed adaptive integrated-sliding-mode controller synthesis for string stability of vehicle platoons," IEEE Transactions on Intelligent Transportation Systems, vol. 17, no. 9, pp. 24192429, 2016.

[16] L. Zuo, R. Cui, and W. Yan, "Terminal sliding mode-based cooperative tracking control for non-linear dynamic systems," Transactions of the Institute of Measurement and Control, vol. 39, no. 7, pp. 1081-1087, 2017.
[17] J.-C. Song and Y.-F. Ju, "Distributed adaptive sliding mode control for vehicle platoon with uncertain driving resistance and actuator saturation," Complexity, vol. 2020, no. 20, pp. 1-12, Article ID 7581517, 2020.

[18] G. Rodonyi, "An adaptive spacing policy guaranteeing string stability in multi-brand ad hoc platoons," IEEE Transactions on Intelligent Transportation Systems, vol. 19, no. 6, pp. 1902-1912, 2018.

[19] D. Swaroop, J. K. Hedrick, and S. B. Choi, "Direct adaptive longitudinal control of vehicle platoons," IEEE Transactions on Vehicular Technology, vol. 50, no. 1, pp. 150-161, 2001.

[20] B. Bayar, S. A. Sajadi-Alamdari, F. Viti, and H. Voos, "Impact of different spacing policies for adaptive cruise control on traffic and energy consumption of electric vehicles," in Proceedings of the 2016 24th Mediterranean Conference on Control and Automation (MED), pp. 1349-1354, IEEE, Valletta, Malta, July 2016.

[21] J. Zhang and P. Ioannou, "Longitudinal control of heavy trucks in mixed traffic: environmental and fuel economy considerations," IEEE Transactions on Intelligent Transportation Systems, vol. 7, no. 1, pp. 92-104, 2006.

[22] T. Petrinić and I. Petrović, "Longitudinal spacing control of vehicles in a platoon for stable and increased traffic flow," in Proceedings of the 2012 IEEE International Conference on Control Applications, pp. 178-183, IEEE, Dubrovnik, Croatia, October 2012.

[23] H. E. Sungu, M. Inoue, and J. I. Imura, "Nonlinear spacing policy based vehicle platoon control for local string stability and global traffic flow stability," in Proceedings of the 2015 European Control Conference, pp. 3396-3401, ECC), Linz, Austria, July 2015.

[24] G. Guo and W. Yue, "Hierarchical platoon control with heterogeneous information feedback," IET Control Theory \& Applications, vol. 5, no. 15, pp. 1766-1781, 2011.

[25] X. Yu, G. Guo, and H. Lei, "Longitudinal cooperative control for a bidirectional platoon of vehicles with constant time headway policy," in Proceedings of the 2018 Chinese Control and Decision Conference (CCDC), pp. 2427-2432, IEEE, Shenyang, China, June 2018.

[26] J.-W. Kwon and D. Chwa, "Adaptive bidirectional platoon control using a coupled sliding mode control method," IEEE Transactions on Intelligent Transportation Systems, vol. 15, no. 5, pp. 2040-2048, 2014.

[27] H. K. Khalil, "Nonlinear Systems," 2002.

[28] S. P. Bhat and D. S. Bernstein, "Continuous finite-time stabilization of the translational and rotational double integrators," IEEE Transactions on Automatic Control, vol. 43, no. 5, pp. 678-682, 1998.

[29] S. Li, K. Li, R. Rajamani, and J. Wang, "Model predictive multi-objective vehicular adaptive cruise control," IEEE Transactions on Control Systems Technology, vol. 19, no. 3, pp. 556-566, 2011. 